\title{
Joint effect of longevity-associated mitochondrial DNA 5178 C/A polymorphism and alcohol consumption on risk of hyper-LDL cholesterolemia in middle-aged Japanese men
}

Teruyoshi Kawamoto ${ }^{1}$, Akatsuki Kokaze ${ }^{1,2^{*}}$, Mamoru Ishikawa ${ }^{2,3}$, Naomi Matsunaga $^{2}$, Kanae Karita ${ }^{2}$, Masao Yoshida², Naoki Shimada', Tadahiro Ohtsu', Takako Shirasawa', Hirotaka Ochiai', Taku Ito', Hiromi Hoshino ${ }^{1}$ and Yutaka Takashima

\begin{abstract}
Background: Combined effects between mitochondrial DNA 5178 (Mt5178) C/A polymorphism and alcohol consumption on the risk of hypertension or hyperuricemia have been reported. The objective of this study was to investigate whether Mt5178 C/A polymorphism modulates the effects of alcohol consumption on the risk of dyslipidemia.

Methods: A total of 394 male subjects were selected from among individuals visiting the hospital for regular medical check-ups. After Mt5178 C/A genotyping, a cross-sectional study assessing the combined effect of Mt5178 polymorphism and alcohol consumption on the risk of dyslipidemia was conducted.

Results: For men with Mt5178C, alcohol consumption was significantly and negatively associated with the risk of hyper-low-density lipoprotein (LDL) cholesterolemia (serum LDL cholesterol $\geq 140 \mathrm{mg} / \mathrm{dl}$ ) $(P$ for trend $=0.015$ ). After adjustment for age, body mass index (BMI), habitual smoking, coffee consumption and use of antihypertensive medicine, the odds ratio (OR) for hyper-LDL cholesterolemia was significantly lower in daily drinkers with $\mathrm{Mt5178C}$ than non-drinkers with $\mathrm{Mt5178C}(\mathrm{OR}=0.360,95 \%$ confidence intervals: 0.153-0.847). A significant and negative association between alcohol consumption and serum LDL cholesterol levels was also observed in Mt5178C genotypic men ( $P$ for trend < 0.01). On the other hand, the association between Mt5178A genotype and risk of hyper-LDL cholesterolemia does not appear to depend on alcohol consumption.

Conclusions: For Mt5178C genotypic men, alcohol consumption may reduce the risk of hyper-LDL cholesterolemia.
\end{abstract}

\section{Introduction}

Mitochondrial DNA 5178 (Mt5178) C/A polymorphism, also known as NADH dehydrogenase subunit-2 237 (ND2-237) Leu/Met, is a longevity-associated mitochondrial DNA polymorphism [1-3]. The frequency of the Mt5178A genotype is significantly higher in Japanese centenarians than in the general population [1]. Japanese individuals with Mt5178A are more resistant to lifestyle-

\footnotetext{
* Correspondence: akokaze@med.showa-u.ac.jp

'Department of Public Health, Showa University School of Medicine, 1-5-8

Hatanodai, Shinagawa-ku, Tokyo 142-8555, Japan

Full list of author information is available at the end of the article
}

related adult-onset diseases, such as hypertension [4], diabetes [5], myocardial infarction [6,7] and cerebrovascular disorders [8], than those with Mt5178C. This polymorphism is also associated with serum lipid levels [9] and fasting plasma glucose levels [10].

A combined effect of Mt5178 C/A polymorphism and alcohol consumption on serum lipid levels has been reported [11]. Alcohol consumption is negatively associated with serum triglyceride levels in healthy male subjects with Mt5178A and with serum low-density lipoprotein (LDL) cholesterol levels in those with Mt5178C [11]. Previous studies have reported the joint

\section{Ciomed Central}


effects of Mt5178 C/A polymorphism and alcohol intake on the risk of hypertension or hyperuricemia in middleaged Japanese men. For men with the Mt5178C genotype, daily alcohol consumption may increase the risk of hypertension [4] or hyperuricemia [12]. However, there have been no reports on the association between Mt5178 C/A polymorphism and alcohol intake on the risk of dyslipidemia, which is clinically important for the prevention of atherosclerotic diseases [13].

The objective of this study was to investigate whether there is a joint effect of Mt5178 C/A polymorphism and alcohol consumption on the risk of dyslipidemia in middle-aged Japanese male subjects.

\section{Materials and methods Study subjects}

Participants were recruited from among individuals visiting the Mito Red Cross Hospital for regular medical check-ups between August 1999 and August 2000. This study was conducted in accordance with the Declaration of Helsinki and was approved by the Ethics Committee of the Kyorin University School of Medicine. Written informed consent was obtained from 602 volunteers before participation. Because the number of women was insufficient for classification into groups based on Mt5178 C/A genotype and alcohol consumption habits, women were excluded. Patients taking antihyperlipidemic medication were excluded, and diabetic patients were also excluded because of their higher prevalence of dyslipidemia when compared to non-diabetic patients [14]. Thus, 406 men, including patients using antihypertensive medication, were enrolled in the study. Twelve individuals with unclear data were also excluded. Therefore, subjects comprised 394 Japanese men (age, $53.9 \pm$ 7.9 years; mean $\pm \mathrm{SD}$ ).

\section{Clinical characteristics of subjects}

Determination of blood chemical and physical data was conducted as described previously [9]. Serum total cholesterol levels, serum high-density lipoprotein (HDL) cholesterol levels, serum triglyceride levels, fasting plasma glucose levels and serum uric acid levels were measured using standard laboratory techniques. LDL cholesterol levels were calculated by Friedewald's formula [15]. Dyslipidemias were defined according to the Japan Atherosclerosis Society guidelines for the prevention of atherosclerotic cardiovascular diseases (hyperLDL cholesterolemia was defined as serum LDL cholesterol $\geq 140 \mathrm{mg} / \mathrm{dl}$, hypo-HDL cholesterolemia was defined as serum HDL cholesterol $<40 \mathrm{mg} / \mathrm{dl}$ and hypertriglyceridemia was defined as serum triglyceride $\geq$ $150 \mathrm{mg} / \mathrm{dl}$ ) [13]. The values presented for both systolic blood pressure and diastolic blood pressure were the averages of two measurements obtained by physicians.
Body mass index (BMI) was defined as the ratio of subject weight $(\mathrm{kg})$ to the square of subject height $(\mathrm{m})$. A survey of alcohol consumption, habitual smoking, coffee intake and use of antihypertensive medication was performed by means of questionnaire. Alcohol consumption was classified based on drinking frequency (daily drinkers; occasional drinkers, which include those who drink several times per week or per month; and non- or ex-drinkers). Habitual smoking was classified as non- or ex-smokers and current smokers. Coffee consumption was classified based on number of cups of coffee per day (less than one cup per day; one, two or three cups per day; and more than four cups per day). For use of antihypertensive medication, subjects were classified as taking no drug treatment or taking medicine.

\section{Genotyping}

DNA was extracted from white blood cells using the DNA Extractor WB kit (Wako Pure Chemical Industries, Japan). The Mt5178 C/A polymorphism was detected by polymerase chain reaction (PCR) and digestion with AluI restriction enzyme. Primers were: forward 5'-CTTAG CAT ACTCCTCAATTACCC-3'; and reverse 5'-GTGAA TTCTTCGATAATGGCCCA-3'. PCR was performed with $50 \mathrm{ng}$ of genomic DNA in buffer containing 0.2 $\mu \mathrm{mol} / \mathrm{l}$ each primer, $1.25 \mathrm{mmol} / \mathrm{l} \mathrm{dNTPs}, 1.5 \mathrm{mmol} / \mathrm{l}$ $\mathrm{MgCl}_{2}$, and $1 \mathrm{U}$ of Taq DNA polymerase. After an initial denaturation at $94^{\circ} \mathrm{C}$ for $5 \mathrm{~min}, \mathrm{PCR}$ was conducted through 40 cycles as follows: denaturation at $94^{\circ} \mathrm{C}$ for $30 \mathrm{~s}$; annealing at $60^{\circ} \mathrm{C}$ for $60 \mathrm{~s}$; and polymerase extension at $72^{\circ} \mathrm{C}$ for $90 \mathrm{~s}$. After cycling, a final extension at $72^{\circ} \mathrm{C}$ for 10 min was performed. PCR products were digested with $A l u I$ restriction enzyme (Nippon Gene, Japan) at $37^{\circ} \mathrm{C}$ overnight, and were electrophoresed on $1.5 \%$ agarose gels stained with ethidium bromide for visualization under ultraviolet light. The absence of an AluI site was designated as Mt5178A (279-bp fragment), and the presence of this restriction site was designated as Mt5178C (175-bp and 104-bp fragments).

\section{Statistical analyses}

Statistical analyses were performed using SAS statistical software, version 9.1, for Windows (SAS Institute, Inc., Cary, NC, 2002). Multiple logistic regression analysis was used to calculate the odds ratio (OR) of dyslipidemia. Differences in serum lipid levels between groups by alcohol consumption were evaluated using the least square means calculated from analysis of covariance. Differences with $P$ values of less than 0.05 were considered to be statistically significant.

\section{Results}

No significant differences in biophysical or biochemical characteristics, or in frequency of dyslipidemia were 
observed between the Mt5178C and Mt5178A genotypes (Table 1).

Significant and negative associations between alcohol consumption and the risk of hyper-LDL cholesterol were observed in Mt5178C genotypic men ( $P$ for trend $=0.015$ and adjusted $P$ for trend $=0.014$, respectively). The OR for hyper-LDL cholesterolemia was significantly lower in daily drinkers with $\mathrm{Mt} 5178 \mathrm{C}$ than in non-drinkers with Mt5178C $(\mathrm{OR}=0.408,95 \%$ confidence intervals (CI): $0.188-0.888, P=0.024$ ) (Table 2 ). After adjustment for age, body mass index, habitual smoking, coffee consumption and use of antihypertensive medication, a significant $\mathrm{OR}$ remained $(\mathrm{OR}=0.360,95 \% \mathrm{CI}$ : $0.153-0.847, P=0.019)$. On the other hand, the association between Mt5178A genotype and hyper-LDL cholesterolemia does not appear to depend on alcohol consumption. No significant joint effect of Mt5178 C/A polymorphism and alcohol consumption on risk of hypo-HDL cholesterolemia or hypertrygliceridemia was observed (data not shown).

Bonferroni correction for multiple comparisons revealed that serum LDL levels were significantly lower in daily drinkers with Mt5178C than in non- or ex-drinkers with Mt5178C $(P=0.011)$ (Table 3$)$. After adjusting for age and BMI, serum LDL cholesterol levels were also significantly lower in daily drinkers with Mt5178C than in non- or ex-drinkers with Mt5178C $(P=0.007)$. Moreover, after adjusting for age, BMI, habitual smoking, coffee consumption and use of antihypertensive medicine, serum LDL cholesterol levels were significantly lower in occasional drinkers with Mt5178C or daily drinkers with Mt5178C than in non- or ex-drinkers with $\mathrm{Mt5178C}(P=0.049$ and $P=0.008$, respectively). As crude and adjusted $P$-values for trends reached statistically significant levels $(P$ for trend $<$ 0.01 ), a significant and negative association between alcohol consumption and serum LDL cholesterol levels was confirmed in subjects with Mt5178C genotype. On the other hand, no noticeable relationship between alcohol intake and serum LDL cholesterol levels was observed in those with the Mt5178A genotype.

\section{Discussion}

The present study confirmed a novel gene-environment interaction. Longevity-associated Mt5178 C/A polymorphism and alcohol consumption may combine to modify the risk of hyper-LDL cholesterolemia in middle-aged Japanese men. For men with Mt5178C, habitual alcohol consumption may reduce serum LDL cholesterol levels or risk of hyper-LDL cholesterolemia. However, for those with Mt5178A, alcohol consumption does not appear to influence the risk of hyper-LDL cholesterolemia.

Although our previous analysis was carried out from the viewpoint of gerontology [11], this analysis of geneenvironment interactions was undertaken to evaluate the risk of dyslipidemia, which is clinically and preventively important. Therefore, the effect of alcohol on

Table 1 Clinical characteristics of study subjects by Mt5178 C/A genotype

\begin{tabular}{llll}
\hline & Mt5178C & Mt5178A & P value \\
\hline & $\mathbf{N}=\mathbf{2 3 9}$ & $\mathbf{1 5 5}$ \\
\cline { 2 - 4 } Age (y) & $54.3 \pm 7.8$ & $53.2 \pm 7.8$ & 0.171 \\
Body Mass Index (kg/m²) & $23.3 \pm 2.8$ & $23.5 \pm 2.6$ & 0.461 \\
Systolic blood pressure (mmHg) & $125.8 \pm 15.9$ & $125.7 \pm 14.1$ & 0.940 \\
Diastolic blood pressure (mmHg) & $74.0 \pm 10.7$ & $73.8 \pm 9.1$ & 0.829 \\
Total cholesterol (mg/dl) & $203.5 \pm 34.3$ & $201.9 \pm 31.8$ & 0.672 \\
LDL cholesterol (mg/dl) & $121.6 \pm 34.8$ & $117.9 \pm 30.5$ & 0.281 \\
HDL cholesterol (mg/dl) & $54.6 \pm 13.6$ & $56.3 \pm 16.2$ & 0.269 \\
Triglyceride (mg/dl) & $136.7 \pm 91.1$ & $139.5 \pm 90.8$ & 0.766 \\
Fasting plasma glucose (mg/dl) & $97.2 \pm 9.4$ & $97.6 \pm 9.7$ & 0.672 \\
Uric acid (mg/dl) & $5.98 \pm 1.21$ & $5.92 \pm 1.21$ & 0.673 \\
Alcohol consumption (daily/occasional/non- or ex-) (\%) & $46.4 / 35.2 / 18.4$ & $47.7 / 38.7 / 13.6$ \\
Coffee consumption (< 1 cup per day/1-3 cups per day/z 4 cups per day) (\%) & $44.8 / 46.0 / 9.2$ & $36.8 / 51.6 / 11.6$ & 0.426 \\
Current smokers (\%) & 41.4 & 40.7 & 0.274 \\
Antihypertensive medication use (\%) & 18.8 & 12.9 & 0.878 \\
Hyper-LDL cholesterolemia (\%) & 26.4 & 25.8 & 0.122 \\
Hypo-HDL cholesterolemia (\%) & 11.7 & 12.3 & 0.903 \\
Hypertriglyceridemia (\%) & 29.7 & 29.7 & 0.871 \\
\hline
\end{tabular}

$\mathrm{LDL}$; low-density lipoprotein, HDL; high-density lipoprotein. Age, body mass index, systolic blood pressure, diastolic blood pressure, serum total cholesterol levels, serum LDL cholesterol levels, serum HDL cholesterol levels, serum triglyceride levels, fasting plasma glucose levels and serum uric acid levels are given as means \pm S.D. All $P$ values depict significance of differences between Mt5178C and Mt5178A. For alcohol consumption, coffee consumption, current smokers, antihypertensive medication use, hyper-LDL cholesterolemia, hypo-HDL cholesterolemia and hypertriglyceridemia, $P$ values were calculated by chi-squared test. 
Table 2 Odds ratios (ORs) and 95\% confidence intervals (Cls) for hyper-LDL cholesterolemia by Mt5178 C/A genotype and alcohol consumption

\begin{tabular}{|c|c|c|c|c|}
\hline \multirow[b]{2}{*}{ Genotype and alcohol consumption } & \multicolumn{2}{|c|}{ Frequency } & \multirow[b]{2}{*}{ OR $(95 \% \mathrm{Cl})$} & \multirow[b]{2}{*}{$\begin{array}{l}\text { Adjusted ORt } \\
(95 \% \mathrm{Cl})\end{array}$} \\
\hline & $\begin{array}{c}\text { Normal LDL cholesterol } \\
\text { (LDL cholesterol }<140 \mathrm{mg} / \mathrm{dl})\end{array}$ & $\begin{array}{l}\text { Hyper-LDL cholesterolemia } \\
\text { (LDL cholesterol } \geq 140 \mathrm{mg} / \mathrm{dl} \text { ) }\end{array}$ & & \\
\hline \multicolumn{5}{|l|}{ Mt5178C } \\
\hline Non- or ex-drinkers (\%) & $28(63.6)$ & $16(36.4)$ & 1 (reference) & 1 (reference) \\
\hline Occasional drinkers (\%) & $58(69.1)$ & $26(30.9)$ & $0.784(0.364-1.692)$ & $0.694(0.290-1.660)$ \\
\hline \multirow[t]{2}{*}{ Daily drinkers (\%) } & $90(81.1)$ & $21(18.9)$ & $0.408(0.188-0.888)^{*}$ & $0.360(0.153-0.847)^{*}$ \\
\hline & & & $P$ for trend $=0.015$ & $P$ for trend $=0.014$ \\
\hline \multicolumn{5}{|l|}{ Mt5178A } \\
\hline Non- or ex-drinkers (\%) & $16(76.2)$ & $5(23.8)$ & 1 (reference) & 1 (reference) \\
\hline Occasional drinkers (\%) & $45(75.0)$ & $15(25.0)$ & $1.067(0.334-3.409)$ & $1.190(0.318-4.447)$ \\
\hline \multirow[t]{2}{*}{ Daily drinkers (\%) } & $54(73.0)$ & $20(27.0)$ & $1.185(0.384-3.660)$ & $1.232(0.361-4.206)$ \\
\hline & & & $P$ for trend $=0.730$ & $P$ for trend $=0.558$ \\
\hline
\end{tabular}

LDL; low-density lipoprotein. $+O R$ adjusted for age, body mass index, habitual smoking, coffee consumption and antihypertensive medication use. ${ }^{*} P<0.05$.

reducing the risk of hyper-LDL cholesterolemia in Mt5178C genotypic men may be more clinically and preventively informative. A previous study reported a significantly negative correlation between alcohol intake and serum LDL cholesterol levels among 193 healthy subjects with Mt5178C [11]. In the present study, among the subjects including patients using antihypertensive medication, alcohol consumption was also significantly and negatively associated with serum LDL cholesterol levels in 239 Mt5178C genotypic men. On multiple logistic regression analysis or analysis of covariance, use of antihypertensive medication was adjusted for.

Individuals with $\mathrm{Mt} 5178 \mathrm{C}$ are reported to be more susceptible to atherosclerotic diseases than those with Mt5178A [6-8]. Our results suggest that, for Mt5178C genotypic men, alcohol intake decreases serum LDL cholesterol levels and reduces the risk of hyper-LDL cholesterolemia, which is a crucial risk factor for coronary heart disease [13]. However, for men with Mt5178C, daily alcohol consumption increases the risk of hypertension [4] and hyperuricemia [12]. From the viewpoint of preventing atherosclerotic diseases, it is difficult to determine whether alcohol intake is beneficial for Mt5178C genotypic men. In contrast, individuals with Mt5178A are genetically more resistant to atherosclerotic diseases than those with Mt5178C. For men with Mt5178A, alcohol intake decreases serum triglyceride levels [11]. However, daily alcohol consumption is positively associated with yearly changes in serum LDL cholesterol levels in men with Mt5178A [16]. It is also unclear whether alcohol consumption is favorable for Mt5178A genotypic men.

Combined effects of Mt5178 C/A polymorphism and coffee consumption on risk factors for atherosclerotic diseases have been reported previously $[17,18]$. For men

Table 3 Serum LDL cholesterol levels in habitual alcohol consumption groups by Mt5178 C/A genotype

\begin{tabular}{|c|c|c|c|c|}
\hline & \multicolumn{3}{|c|}{ Alcohol consumption } & \multirow[t]{2}{*}{$P$ for trend } \\
\hline & Non- or ex-drinkers & Occasional drinkers & Daily drinkers & \\
\hline Mt5178C & $N=44$ & $N=84$ & $N=111$ & \\
\hline LDL cholesterol & $135.0 \pm 5.2$ & $120.5 \pm 3.7$ & $117.1 \pm 3.3^{*}$ & 0.007 \\
\hline LDL cholesterol † & $135.8 \pm 5.2$ & $120.4 \pm 3.8$ & $116.9 \pm 3.3^{* *}$ & 0.005 \\
\hline LDL cholesterol ₹ & $135.6 \pm 6.3$ & $119.5 \pm 4.9^{*}$ & $116.4 \pm 4.1^{* *}$ & 0.006 \\
\hline Mt5178A & $N=21$ & $N=60$ & $N=74$ & \\
\hline LDL cholesterol & $121.4 \pm 6.7$ & $118.5 \pm 4.0$ & $116.5 \pm 3.6$ & 0.506 \\
\hline LDL cholesterol † & $122.1 \pm 6.7$ & $118.4 \pm 3.9$ & $116.3 \pm 3.5$ & 0.445 \\
\hline LDL cholesterol ‡ & $121.4 \pm 7.5$ & $117.6 \pm 5.1$ & $116.1 \pm 4.7$ & 0.502 \\
\hline
\end{tabular}

LDL: low-density lipoprotein. $+\mathrm{LDL}$ cholesterol levels are given as least-square means \pm S.E. adjusted for age and body mass index; $\neq \mathrm{LDL}$ cholesterol levels are given as least-square means \pm S.E. adjusted for age, body mass index, habitual smoking, coffee consumption and antihypertensive medication use. Bonferroni correction for multiple comparisons was applied. ${ }^{*} P<0.05$ vs. Non- or ex-drinkers with Mt5178C; ${ }^{* *} P<0.01$ vs. Non- or ex-drinkers with Mt5178C. 
with Mt5178C, coffee consumption appears to reduce the risk of hypertension [17] and abnormal glucose tolerance [18], and may thus reduce the risk of atherosclerotic diseases. Whereas coffee intake appears to provide antiatherogenic benefits for Mt5178C genotypic men, for Mt5178A genotypic men, coffee consumption appears to increase the risk of hyper-LDL cholesterolemia [19]. Using genotyping of Mt5178 C/A, integration of information regarding alcohol or coffee consumption may contribute to personalized prevention of life-threatening cardiovascular or cerebrovascular diseases.

Although the association between increasing alcohol intake and decreasing serum LDL cholesterol levels is not apparently modified by alcohol dehydrogenase genotype [20], Corella et al. reported that, among men with apolipoprotein E gene (APOE) $\varepsilon 2$, serum LDL cholesterol levels were significantly lower in drinkers than in non-drinkers [21]. In addition to APOE $\varepsilon 2 / \varepsilon 3 / \varepsilon 4$ allele types, several polymorphisms, such as apolipoprotein A5 gene polymorphism [22], apolipoprotein C-III gene polymorphism [23], peroxisome proliferator-activated receptor $\alpha$ gene polymorphism [24], cholesteryl ester transfer protein Taq1B polymorphism [25] and promoter polymorphism of hepatic lipase gene [26], influence the effects of alcohol consumption on serum LDL cholesterol concentrations. Therefore, gene-gene-environment or gene-gene-gene-environment interactions with serum LDL cholesterol levels should be investigated. Further genetic epidemiological investigations will establish individualized alcohol drinking habits in terms of optimizing serum LDL cholesterol concentrations.

The mechanisms underlying the joint effects of Mt5178 C/A (ND2-237 Leu/Met) polymorphism and alcohol consumption on the risk of hyper-LDL cholesterolemia remain unknown. They presumably depend on the amino-acid-related biochemical differences in NADH dehydrogenase in response to alcohol between ND2-237Leu and ND2-237Met. In any case, clarification of the mechanisms of the combined effects of Mt5178 C/A (ND2-237 Leu/Met) polymorphism and alcohol consumption on serum LDL cholesterol concentrations remains a matter for further molecular biological investigation.

Chi-squared test did not reveal a significant difference in the frequency of Mt5178A between this study and other molecular epidemiological studies [27], thus suggesting that there is no genetic bias in the subjects in this study. However, there may have been selection bias due to the recruiting of subjects from among those visiting the hospital for regular medical check-ups. Moreover, the sample size was not sufficient to test the research hypothesis. Although cross-sectional studies can provide a suggestive causality, they cannot establish solid causal links. Therefore, in order to resolve these problems, a large-scale population-based follow-up study is necessary.

Another limitation of this study was the evaluation of habitual alcohol intake based on the frequency of alcohol consumption. As there is a J-shaped curve for the relationship between volume of alcohol consumption and risk of coronary heart disease [28] or ischemic stroke [29], evaluation of volume of alcohol consumption is of great import. Although we have also used this evaluation in previous studies $[4,11,12,16,30]$, whether there is any joint effect between Mt5178 C/A polymorphism and volume of alcohol intake on the risk of hyper-LDL cholesterolemia warrants further investigation. Männistö et al. reported that the percentage of energy from fat was significantly lower in male abstainers than in male alcohol drinkers [31]. Therefore, dietary information is required in order to precisely evaluate the relationship between alcohol consumption and serum LDL cholesterol concentration. In this study, LDL cholesterol levels were calculated by means of Friedewald's formula [15]. Therefore, direct measurement of serum LDL cholesterol levels is desirable.

In conclusion, a joint effect of longevity-associated Mt5178 C/A polymorphism and habitual alcohol consumption on the risk of hyper-LDL cholesterolemia was observed in middle-aged Japanese men. For men with $\mathrm{Mt} 5178 \mathrm{C}$, alcohol intake may reduce the risk of hyperLDL cholesterolemia. Considering that daily alcohol consumption increases other risk factors of atherosclerotic diseases for Mt5178C genotypic men [4,12], it is difficult to determine whether habitual drinking is preventively beneficial. Although further investigation will be required to establish the individualized optimum for maximizing the risk reduction and minimizing the risk increase of habitual alcohol drinking for atherosclerotic diseases, this information regarding the Mt5178 C/A polymorphism will probably contribute to personalized prevention for cardiovascular or cerebrovascular events.

\section{Acknowledgements}

This study was supported in part by Grants-in-Aid from the Ministry of Education, Culture, Sports, Science and Technology of Japan (No. 14570355, No. 18590572 and No. 23500859) and the Chiyoda Mutual Life Foundation.

\section{Author details}

${ }^{1}$ Department of Public Health, Showa University School of Medicine, 1-5-8 Hatanodai, Shinagawa-ku, Tokyo 142-8555, Japan. ${ }^{2}$ Department of Public Health, Kyorin University School of Medicine, 6-20-2 Shinkawa, Mitaka-shi, Tokyo 181-8611, Japan. ${ }^{3}$ Mito Red Cross Hospital, 3-12-48 Sannomaru, Mitoshi, Ibaraki 310-0011, Japan.

\section{Authors' contributions}

TK analyzed the data and drafted the manuscript; AK designed the study, carried out the epidemiological survey, carried out the genotyping, analyzed the data, and drafted the manuscript; MI collected the samples; NM helped to carry out the genotyping; KK and MY carried out the epidemiological 
survey; NS, TO, TS, HO, TI, and $\mathrm{HH}$ assisted in data analysis and helped with interpreting the results; YT designed the study and carried out the epidemiological survey. All authors have read and approved the final manuscript.

\section{Competing interests}

The authors declare that they have no competing interests.

Received: 20 May 2011 Accepted: 25 June 2011 Published: 25 June 2011

\section{References}

1. Tanaka M, Gong JS, Zhang J, Yoneda M, Yagi K: Mitochondrial genotype associated with longevity. Lancet 1998, 351:185-186.

2. Alexe G, Fuku E, Bilal E, Ueno $H$, Nishigaki $Y$, Fujita $Y$, Ito $M$, Arai $Y$, Hirose $N$, Bhanot G, Tanaka M: Enrichment of longevity phenotype in mtDNA haplogroups D4b2b, D4a, and D5 in the Japanese population. Hum Genet 2007, 121:347-356.

3. Bilal E, Rabadan R, Alexe G, Fuku N, Ueno H, Nishigaki $Y$, Fujita $Y$, Ito M, Arai $Y$, Hirose N, Ruckenstein A, Bhanot G, Tanaka M: Mitochondrial DNA haplogroup D4a is a marker for extreme longevity in Japan. PLOS One 2008, 3:e2421.

4. Kokaze A, Ishikawa M, Matsunaga N, Yoshida M, Satoh M, Teruya K, Masuda Y, Honmyo R, Uchida Y, Takashima Y: NADH dehydrogenase subunit-2 237 Leu/Met polymorphism modifies the effects of alcohol consumption on risk for hypertension in middle-aged Japanese men. Hypertens Res 2007, 30:213-218.

5. Wang D, Taniyama M, Suzuki $Y$, Katagiri T, Ban Y: Association of the mitochondrial DNA $5178 \mathrm{~A} / \mathrm{C}$ polymorphism with maternal inheritance and onset of type 2 diabetes in Japanese patients. Exp Clin Endocrinol Diabetes 2001, 109:361-364.

6. Mukae S, Aoki S, Itoh S, Sato R, Nishio K, Iwata T, Katagiri T: Mitochondrial $5178 \mathrm{~A} / \mathrm{C}$ genotype is associated with acute myocardial infarction. Circ $J$ 2003, 67:16-20.

7. Takagi K, Yamada Y, Gong JS, Sone T, Yokota M, Tanaka M: Association of a 5178C『A (Leu237Met) polymorphism in the mitochondrial DNA with a low prevalence of myocardial infarction in Japanese individuals. Atherosclerosis 2004, 175:281-286.

8. Ohkubo R, Nakagawa M, Ikeda K, Kodama T, Arimura K, Akiba S, Saito M, Ookatsu Y, Atsuchi Y, Yamano Y, Osame M: Cerebrovascular disorders and genetic polymorphisms: mitochondrial DNA5178C is predominant in cerebrovascular disorders. J Neurol Sci 2002, 198:31-35.

9. Kokaze A, Ishikawa M, Matsunaga N, Yoshida M, Sekine Y, Teruya K Takeda N, Sumiya Y, Uchida Y, Takashima Y: Association of the mitochondrial DNA $5178 \mathrm{~A} / \mathrm{C}$ polymorphism with serum lipid levels in the Japanese population. Hum Genet 2001, 109:521-525.

10. Kokaze A, Ishikawa M, Matsunaga N, Yoshida M, Makita R, Satoh M, Teruya K, Sekiguchi K, Masuda Y, Harada M, Uchida Y, Takashima Y: Longevity-associated mitochondrial DNA $5178 \mathrm{C} / \mathrm{A}$ polymorphism is associated with fasting plasma glucose levels and glucose tolerance in Japanese men. Mitochondrion 2005, 5:418-425

11. Kokaze A, Ishikawa M, Matsunaga N, Yoshida M, Sekine $Y$, Sekiguchi $K$, Satoh M, Harada M, Teruya K, Takeda N, Uchida Y, Tsunoda T, Takashima Y: Longevity-associated mitochondrial DNA 5178 A/C polymorphism modulates effects of daily drinking and cigarette consumption on serum triglyceride levels in middle-aged Japanese men. Exp Gerontol 2003, 38:1071-1076.

12. Kokaze A, Ishikawa M, Matsunaga N, Yoshida M, Satoh M, Teruya K, Honmyo R, Yorimitsu M, Masuda Y, Uchida Y, Takashima Y: Longevityassociated NADH dehydrogenase subunit-2 237 Leu/Met polymorphism influences the effects of alcohol consumption on serum uric acid levels in nonobese Japanese men. J Hum Genet 2006, 51:765-771.

13. Japan Atherosclerosis Society: Japan Atherosclerotic Society (JAS) guidelines for prevention of atherosclerotic cardiovascular diseases. Tokyo: Kyowa Kikaku; 2007.

14. Yoshino G, Hirano T, Kazumi T: Dyslipidemia in diabetes mellitus. Diabetes Res Clin Pract 1996, 33:1-14

15. Friedewald WT, Levy RI, Fredrickson DS: Estimation of the concentration of low-density lipoprotein cholesterol in plasma, without use of the preparative ultracentrifuge. Clin Chem 1972, 18:499-502.

16. Makita R, Kokaze A, Ohtsu T, Ishikawa M, Matsunaga N, Karita K, Yoshida M, Tanaka N, Yamamoto M, Hayashi J, Takashima Y, Kitamoto K: Longevity- associated NADH dehydrogenase subunit-2 237 Leu/Met polymorphism modulates the effects of daily alcohol drinking on yearly changes in serum total and LDL cholesterol in Japanese men. Acta Med Okayama 2009, 63:331-338.

17. Kokaze A, Ishikawa M, Matsunaga N, Karita K, Yoshida M, Ohtsu T, Shirasawa T, Sekii H, Ito T, Kawamoto T, Takashima Y: NADH dehydrogenase subunit-2 237 Leu/Met polymorphism modulates the effects of coffee consumption on the risk of hypertension in middleaged Japanese men. J Epidemiol 2009, 19:231-236.

18. Kokaze A, Ishikawa M, Matsunaga N, Karita K, Yoshida M, Ohtsu T, Shirasawa T, Haseba Y, Satoh M, Teruya K, Hoshino H, Takashima Y: Longevity-associated mitochondrial DNA 5178 C/A polymorphism modifies the effect of coffee consumption on glucose tolerance in middle-aged Japanese men. In Handbook on longevity: genetics, diet and disease. Edited by: Bentely JV, Keller MA. New York: Nova Science Publishers; 2009:139-160.

19. Kokaze A, Ishikawa M, Matsunaga N, Karita K, Yoshida M, Shimada N, Ohtsu T, Shirasawa T, Ochiai H, Kawamoto T, Ito T, Hoshino H, Takashima Y: Combined effect of longevity-associated mitochondrial DNA 5178 C/A polymorphism and coffee consumption on the risk of hyper-LDL cholesterolemia in middle-aged Japanese men. J Hum Genet 2010, 55:577-581.

20. Tolstrup JS, Grønbaek M, Nordestgaard BG: Alcohol intake, myocardial infarction, biochemical risk factors, and alcohol dehydrogenase genotypes. Circ Cardiovasc Genet 2009, 2:507-514.

21. Corella D, Tucker K, Lahoz C, Coltell O, Cupples LA, Wilson WF, Shaefer EJ, Ordovas JM: Alcohol drinking determines the effect of the APOE locus on LDL-cholesterol concentration in men: the Framingham Offspring Study. Am J Clin Nutr 2001, 73:736-745.

22. Yin RX, Li YY, Liu WY, Zhang L, Wu JZ: Interactions of the apolipoprotein A5 gene polymorphisms and alcohol consumption on serum lipid levels. Plos One 2011, 6:e17954.

23. Ruixing $Y$, Yiyang $L$, Meng $L$, Kela $L$, Xingjiang $L$, Lin $Z$, Wanying $L$, Jinzhen W, Dezhai $Y$, Weixiong L: Interactions of the apolipoprotein C-III $3238 \mathrm{C}>\mathrm{G}$ polymorphism and alcohol consumption on serum triglyceride levels. Lipids Health Dis 2010, 9:86.

24. Naito H, Kamijima M, Yamanoshita O, Nakahara A, Katoh T, Tanaka N, Aoyama T, Gonzalez FJ, Nakajima T: Differential effects of aging, drinking and exercise on serum cholesterol levels dependent on the PPERAV227A polymorphism. J Occup Health 2007, 49:353-362.

25. Zhou Y, Yin R, Deng Y, Li Y, Wu J: Interactions between alcohol intake and the polymorphism of rs708272 on serum high-density lipoprotein cholesterol levels in the Guangxi Hei Yi Zhuang population. Alcohol 2008, 42:583-591.

26. Meng $L$, Ruixing $Y$, Yiyang $L$, Xingiiang $L$, Kela $L$, Wanying $L$, Lin $Z$, Weixiong $L$, Dezhai $Y$, Shangling P: Association of LIPC -250G > A polymorphism and several environmental factors with serum lipid levels in the Guangxi Bai Ku Yao and Han populations. Lipids Health Dis 2010, 9:28.

27. Shimokata H, Yamada Y, Nakagawa M, Okubo R, Saido T, Funakoshi A, Miyasaka K, Ohta S, Tsujimoto G, Tanaka M, Ando F, Niino N: Distribution of geriatric disease-related genotypes in the National Institute for Longevity Sciences, Longevity Study of Aging (NILS-LSA). J Epidemiol 2000, 10:S46-S55.

28. Corrao G, Bagnardi V, Zambon A, La Vecchia C: A meta-analysis of alcohol consumption and the risk of 15 diseases. Prev Med 2004, 38:613-619.

29. Reynolds K, Lewis B, Nolen JD, Kinney GL, Sathya B, He J: Alcohol consumption and risk of stroke: a meta-analysis. JAMA 2003, 289:579-588.

30. Kokaze A, Ishikawa M, Matsunaga N, Yoshida M, Sekine Y, Sekiguchi $K$, Harada M, Satoh M, Teruya K, Takeda N, Fukazawa S, Uchida Y, Takashima Y: Longevity-associated mitochondrial DNA $5178 \mathrm{~A} / \mathrm{C}$ polymorphism and blood pressure in the Japanese population. J Hum Hypertens 2004, 18:41-45.

31. Männistö $S$, Uusitalo K, Roos E, Fogelholm M, Pietinen P: Alcohol beverage drinking, diet and body mass index in a cross-sectional survey. Eur J Clin Nutr 1997, 51:326-332.

\section{doi:10.1186/1476-511X-10-105}

Cite this article as: Kawamoto et al.: Joint effect of longevity-associated mitochondrial DNA $5178 \mathrm{C} / \mathrm{A}$ polymorphism and alcohol consumption on risk of hyper-LDL cholesterolemia in middle-aged Japanese men. Lipids in Health and Disease 2011 10:105. 